\title{
REFLECTION OF MICROWAVES FROM THE MOON.
}

\author{
BY Z. BAY.
}

(RECEIVED I8TH NOVEMBER I946.)

SUMMARY.

The possibility of the reception of microwaves reflected by the Moon is considered theoretically. To increase the signal/noise ratio of the receiver, $i$. e. to raise the signal above noise-level, a special method of cumulation is proposed. The equipment developed according to the above principles is described, and the experimental method and results are discussed.

The experiments show that electromagnetic waves of $2,5 \mathrm{~m}$ wave-length can pass through the ionosphere. The orders of magnitude of the absorption and dispersion in the ionosphere and the reflection coefficient of the Moon's surface are given by the experiment as expected. The success of the Moon experiment adds a most direct evidence to other experiments which confirm the validity of the Copernican view.

It is only recently that the technique of microwaves has enabled Man to leave his terrestrial realm and to make celestial bodies the objects of his experiments. Until now our knowledge of celestial objects and of the Universe has been based only on observations.

The above possibility is utilized in our experiment, in which microwave-signals are sent out towards the Moon, the signals are reflected by it, and return to the Earth where they can be detected and measured.

I first proposed the plan of such a Moon-experiment in March, I944 (see below), and with my co-workers I immediately began calculations which showed that the experiment could be successfully carried out with the apparatus at our disposal, or the ones that could be developed under our scientific and technical conditions if the rôle of some unknown factors can be supposed to be advantageous. Such factors are particularly: penetration of microwaves through the ionosphere and the reflectibility of the Moon's surface.

The theory of the ionosphere and the data on electron density as computed from the reflection of short-waves suggest that short electromagnetic waves (less than a few meters' wavelength) pass through the ionosphere without considerable absorption or reflection. No experimental proof of this statement is, however, known as yet. The original intensity of microwaves of cosmic origin ${ }^{1}$ is unknown, as is the rate of the influence of the ionosphere upon these waves. Both these, and the power of reflection

I K. Jansky: Proc. I. R. E. 21, 1387, (1933). 
of the Moon's surface are the very problems which ought to be solved by carrying out systematic Moon-experiments.

The results of the preliminary calculations were encouraging, and the first realization of the experiments was begun early in 1944 in the Research Laboratory of the United Incandescent Lamp and Electrical Co. Ltd., ("Tungsram") Újpest. In view of the danger of air-raids, the microwave laboratory - dealing with principal problems of radio location was removed by the higher military command to Nógrádverőce, where during the summer of 1944 the first equipment for the Moon-experiment was constructed. Soon after the beginning of systematic measurements the return of the Laboratory to Ujpest in autumn I944 interrupted the experiments and the circumstances of the following period prevented the re-installation of the apparatus until March, I945. At this time, however, the researches had to be interrupted again, due to the dismantling of the Tungsram Works when all our equipment was lost. The construction of completely new equipment was begun - for the third time already - in August, r945, and the work was finished in January, I946. After tuning and regulating various parts of the complicated apparatus, the first successful experiment was carried out on the 6th February, I946.

There was no doubt - though no information was available owing to the isolation caused by the war - that similar experiments may have been initiated by other laboratories too, or may be going on simultaneously elsewhere, because of the principal importance mentioned above. Besides its principal significance, the experiments - in case of success - would open a new field of science, allowing the determination of some physical data of the Moon's surface. Also from some technical points of view it may be a forerunner of several possible ideas (see p. 2I.)

In view of this, it can be understood why we made sacrifices even during the past period of great difficulties and it was not our fault that we were finally preceded by American ${ }^{2}$ and Russian ${ }^{3}$ laboratories by a short time. Following the foreign examples - the first success of the experiment was announced by the Hungarian Radio and the daily press, because the experiments were interesting from the foint of view of the general public as well. On the technical problems of the equipment an account was given in the Hungarian Electrotechnical Association on May 24, I946. The theory and the practical solution of the experiments are explained in the following pages.

2 American radio announcement: Jan. 1946., Technical description: Electronics, April, I946, p. 92. known.

3 An announcement in the daily press. January, 1946. No scientific report 
PRINCIPAL CONSIDERATIONS AND CALCULATIONS.

Calculations ${ }^{4}$ carried out in the theory of terrestrial radar can be directly generalized for the case of the Moon-experiment, if the reflection by the ionosphere is - provisionally - neglected, and plausible suppositions are made as to the reflection coefficient of the Moon. I carried out the calculations, the outlines of which are presented here, in cooperation with Drs. G. Papp, K. Simonyi and E. Istvánffy.

From a transmission of $w_{1} \mathrm{~kW}$ 's power

$$
w_{2}=w_{1} \cdot \omega
$$

reaches the Moon's surface, where $\omega \sim 5 \cdot 10^{-6}$ is the solid angle of the Moon. If the average reflection coefficient of the Moon's surface is $r$, then it radiates during unit time: $w_{3}=r \cdot w \cdot w_{1}$ energy. The spacial distribution of this energy depends on the manner of reflection, whether it is regular or diffuse. Calculations show, that these two extreme cases do not differ much from the point of view of the energy returning to the Earth. Thus we may consider the more simple case, $i$. e. the energy reflected by the Moon is distributed in the space uniformly. In this case the field intensity on the Earth's surface is:

$$
E_{1}=10^{5} \cdot \frac{2,12}{D} \sqrt{\dot{w}_{3}} \frac{\mu V}{m}
$$

( $D$ being the Earth-Moon distance in $\mathrm{km}-\mathrm{s}$ ).

If the receiver is connected to a half-wave dipole-antenna and its band-width is $\Omega$, then the field-intensity necessary to get unity signal/noise ratio:

$$
E_{0}=3 \cdot 5 \frac{10^{-3}}{\lambda} \sqrt{\Omega} \mu V / m \text { ( } \lambda: \text { wave length). }
$$

In this computation only the thermal fluctuation voltage (Johnson-effect) of the antenna is considered, which is the ideal condition. In this case the signal/noise ratio of the reception is:

$$
\eta=\frac{E_{1}}{E_{0}}
$$

In our experiments a transmitter of $\lambda=2,5 \mathrm{~m}$ wave-length was available, having an optimum power of $w_{1}=3 \mathrm{~kW}$ (at an impulse of $\tau=6 \cdot 10^{-2}$ sec, duration).

So the intensity of the firld produced by the reflection is: $E_{1}=\sqrt{r}, 2,2 \cdot 10^{-3} \mu V / m, r$ is unknown, but no serious mistake can $\mathrm{b}=$ made in its estimation if $r=\frac{1}{10}$ is taken for the reflection coefficient

4 Z. Bay: Elektrotechnika, vol. 38. pp. I. and 29. (I946). 
of the Moon's crust, corresponding to an average dielectric coefficient $\varepsilon \sim \frac{1}{4}$.

The band-width of the receiver has been $\Omega=2 ; 10^{5} \mathrm{c}$.p.s., so that in the most favourable case: $E_{0}=0,63 \mu \mathrm{V} / \mathrm{m}$. This ideal case is not realized by the receiver, as its noise is caused not only by the antenna, but also by the grid resistances, shot-effect, etc., which altogether can be represented by the noise-figure $\nu$. According to the measurements of Istvánffy and Tari for our set it has been $\nu \sim 3$.

So the signal/noise: ratio of the receiver is $\eta_{1}=\frac{E_{1}}{\nu E_{0}}$, which - using the above data - amounts to $\eta_{1}=3,9 \cdot 10^{-4}$. This extremely low signal/noise ratio rendered a successful experiment hopeless for the time being. It was necessary to look for methods which should essentially enlarge the signal/noise ratio.

By applying a system of antennas (or a reflector) instead of a single antenna, the waves can be directed, $i$. e. energy can be focused. In this way the field intensity can be multiplied by a factor $\varrho$, where $\varrho$ is the gain of the antenna system (reflector). In our case an antenna system of 36 dipoles gave a value $\varrho=9$, by which the signal/noise ratio was improved to

$$
\eta_{2}=\varrho^{2} \cdot \eta_{1}=3,14 \cdot 10^{-2} \text {. }
$$

Further improvement can be obtained by the following reasoning: the aim of the experiment is not the exact measurement of distance, therefore long impulses can be applied, which allow a reduction of band-width, ${ }^{5}$ as shown in the theory of radar.

a) By the analysis of the Fourier-spectrum of the transmitted signal it has been shown that - from the point of view of energy - it is most preferable to choose the length of the transmitted signal $\tau$ equal to the reciprocal of the band width of the receiver $\Omega$.

b) The accuracy of the measurement of time (and therefore that of the distance) is; $\Delta t=\frac{\tau}{\text { signal/noise }}$ i. e. if $\Delta t$ need not be small, $\tau$ may also be large compared to the usual values in radar technique, where $\tau \sim 10^{-5}-10^{-6} \mathrm{sec}:$

Requirements $a$ ) and $b$ ) mean that, in the Moon-experiment, band-width and the length of the transmitted signal can be made about $\Omega \sim 20-30$ c. p. S., $\tau=5 \cdot 10^{-2}$ correspondingly. Using these data the measurement of the distance is not better than. $7500 \mathrm{~km}$ if signal/noise $=\mathrm{I}$, but this does not matter in the present experiment.

z. Bay: Los. cit. p. 3I. 
This reduction of band-width would improve the signal/noise ratio by a factor of about 100 , so obtaining a value of $3,6, i$. e. the reflected signals could be easily detected.

The reduction of the band-width is not a difficult task in itself; it must, however, be considered that in this case the frequency of the transmitter and that of the receiver must agree within $20 \mathrm{c}$. p. s., which is a very high demand. Instead of following this course - the most obvious, but expensive and rather difficult in practice - a method has been chosen, which yields an even greater reduction of band-width, without the necessity of exact tuning of the transmitter and receiver. The principle of this method is, that the reduction of band-width takes place after detection. Using this method, the variation of the transmitter's frequency may cover the receiver's entire frequency-range $\Omega=2,10^{5} \mathrm{c}$. p. s., not making a high demand as to tuning the transmitter and the receiver. The disadvantage of this method is, that - as signal and noise are summed according to the pythagorean rule - the small signal/noise ratio falls with the second power at detection. So $\eta_{2}=3,14 \cdot 10^{-2}$ becomes $\eta_{3}=10^{-3}$. Applying now the reduction of the band-width from $2,10^{5} \mathrm{c}$. p. s. to 2 . Io c. p. s. the signal/noise ratio will be

$$
\eta_{4}=10^{-1}
$$

which is still under the limit of observability.

For this reason a further method had to be looked for, in order to increase the signal/noise ratio by a factor of at least one order of magnitude. For this purpose I proposed a suitable method of cumulation.

The principle of cumulation itself is known in television practice, and applied in the terrestrial radar practice too, where signal/noise ratio is increased by the after-glow of the screen, which amplifies the signal and averages out the noise.

In the case of the Moon-experiment this method of cumulation cannot be used, because the transmitted signals follow after long intervals. The transit time of the signal to the Moon and back is about 2,6 sec. which means that the transmitted signals should follow after longer intervals than this. In our equipment the interval has been chosen as $3 \mathrm{sec}$, i. e. roo impulses take $5 \mathrm{~min}$., rooo impulses take $50 \mathrm{~min}$.

If the period of cumulation is $50 \mathrm{~min}$. the cumulator will preserve the signal for several hours, in order to avoid inaccuracy. For this reason electric condensers cannot be taken into account. It should have very high capacity, and extremely good isolation in order to have a time constant of several hours, which cannot be realized in practice. 
After various considerations I proposed a hydrogen-coulometer, which was succesfully applied in the experiments. The simple design (description s. p. I7.) allows the use of several (ro in our case) coulometers in the experiment, which are connected with the output of the receiver one after the other by a rotating switch. One coulometer is switched on always at one and the same instant after the transmitted signal. In this way ten formerly defined points on the time-scale are recorded, by which - besides the cumulating action - the apparatus also gives a time-diagram of each 3 sec.'s interval. The signal reflected by the Moon, corresponding to its actual distance, falls on one of the coulometers, where they are successively cumulated in the form of quantities of hydrogen gas. The disturbances (voltage fluctuations) are both of positive and negative sign, and are averaged out statistically.

According to the laws of probability the quadratic error occurring on one coulometer after $n$ impulses is proportional to $n$, so the error is $\sqrt{n}$. At the same time the signal on the same coulometer is multiplied by $n$, so the coulometer shows a $\sqrt{n}$ signal/noise ratio instad of the signal/noise ratio of the receiver's output.

By this method - by increasing the total number $n$ of the impulses - any signal can be raised above noise-level, i. e. can be made observable in principle, regardless both of its amplitude and the value of the signal/noise ratio.

This result can be expressed by a phrase usual in radio-practice as follows: the band-width has been reduced to the reciprocal of the total interval, during which a coulometer is switched on. E. g. in case of $n=1000$ impulses: a coulometer is switched on for rooo $.0,06=60$ sec corresponding a band width of $\mathrm{I} / 60 \mathrm{c}$. $\mathrm{p}$. $\mathrm{s}$. By this the signal/noise ratio is increased by a factor of $\sqrt{1000}$, equivalent to a reduction of band-width, or to an increase of the power of the transmitted signal by a factor of rooo.

It is an important advantage of the application of the coulometers that, those which do not receive the reflected signal, continuously show the "zero-reading" of the apparatus, and show the time-integral of the output stage taken during the period of the experiment. Thus intrinsic variations (fluctuations of current, variations of the operational points of the tubes, etc.) of the apparatus are automatically eliminated. This fact can be also expressed by saying that the coulometers, compared to the one receiving the signal, perform a "blind" experiment.

Under these circumstances the possibility of a successful Moonexperiment was guaranteed, as a tolerance of one order of magnitude can be gained by our method. This tolerance was necessary indeed, for we had known that 
I. we cannot increase the transmitted energy,

2. our receiver is not of the high degree of development, which could be arrived at by a further approach to the border of principal possibilities. (Our receivers were only experimental devices, and not technically developed sets),

3. the electrical noise-level inside the town is not favourable for sensitive experiments like this (the coulometer, however, eliminates outer disturbances too),

4. the frequency-constancy of the transmitter during one impulse is not of such a high degre eas could be expected in case of a technically developed equipment.

All these sources of inaccuracy were succesfully eliminated by the cumulating method realized with the aid of coulometers. This, however, has as a consequence, according to the general theory of physical measurements, long periods of observation, that require the apparatus to be in operation for long periods, and make the observation troublesome.

\section{DESCRIPTION OF THE EQUIPMENT.}

Only that equipment is described here, which - as mentioned in the introduction - was developed in the second half of 1945, as it was this by which successful Moon-experiments were carried out. Owing to the grave situation of the Tungsram Works at that time, it seemed almost impossible to build a new equipment at our own cost for the replacement of the one lost. Therefore I applied to the Ministry of Home Defence, which kindly put all the constituent parts left over from the war, and manufactured by the Standard Electrical Co. Ltd. for the purpose of a radar working at $2,5 \mathrm{~m}$ wavelength, at my disposal. ${ }^{6}$ So we could get a transmitter and a receiver set and a power-supply for the latter. It was at this stage that E. Istvanffy, Manager of the Standard El. Co. Ltd. joined our researches, who was in charge of the development of these apparatuses during the war.

It happened on account of these apparatuses that we determined to change the wavelength of our earlier experiments trom $0,5 \mathrm{~m}$ to $2,5 \mathrm{~m}$, after having been shown by the calculations of Istvánffy that $2,5 \mathrm{~m}$ wavelength is not more disadvantageous than $0,5 \mathrm{~m}$ from the point of view of passing through the ionosphere. Istvanffy considered the effect of the ionosphere as a dispersing lens too, and found that this effect cannot reduce the energy density more than by a factor of $12 \%$.

- I should like to take this opportuinty of again expressing my thanks to the Ministry of Home Defence. 
The antenna system, the high-power-supply for the transmitter, the rotating switch, the band-width reducing output stage, the coulometers and relays had to be constructed anew.

The arrangement of the whole equipment is schematically shown on Fig. I. Transmitter, receiver, output stage with measuring instruments

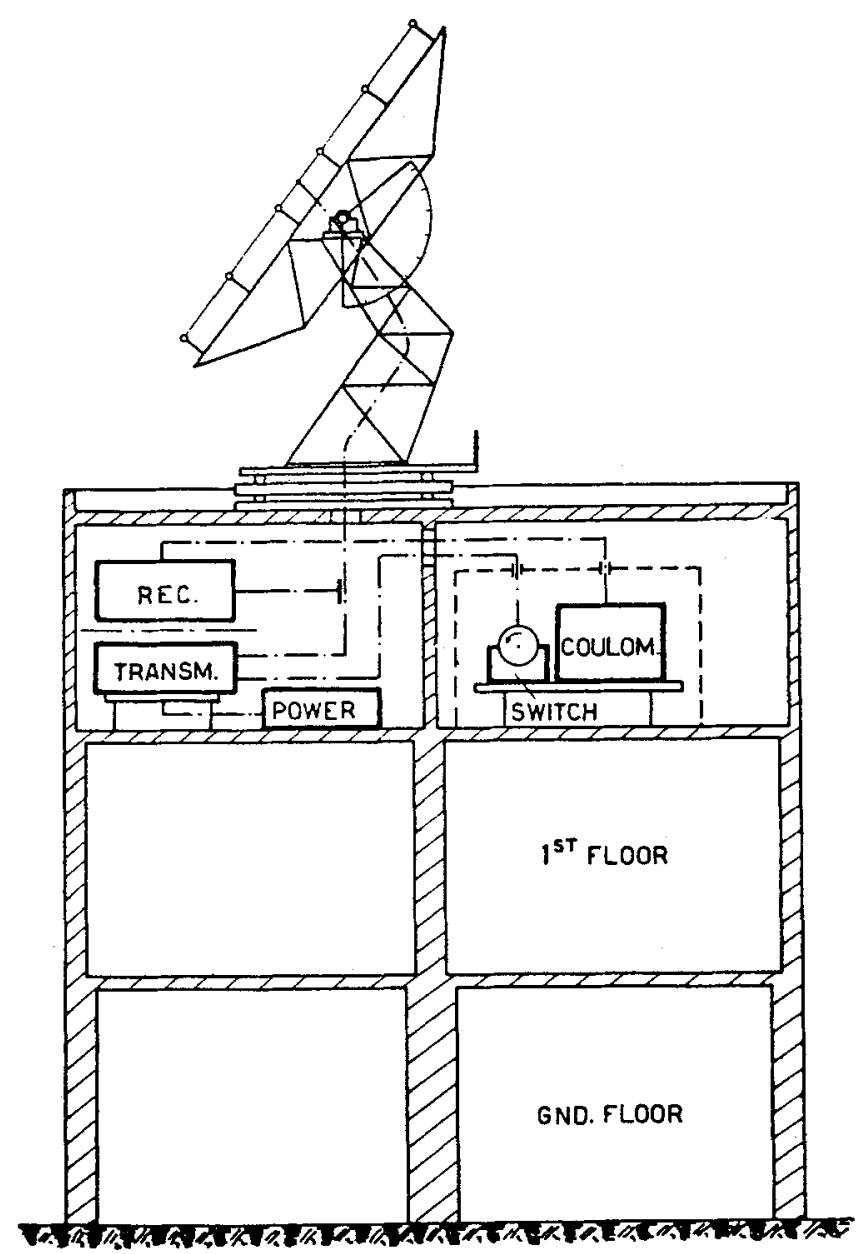

Fig. 1.

were located in two rooms on the second floor of the Tungsram Research Laboratory, the antenna system being situated on the roof, above these rooms.

Fig. 2. shows the block diagram of the whole system. Both the transmitter and the receiver join the transmission line of the antenna system, 
the signals reach the coulometers through the output of the receiver. The periodic operation of the whole system is controlled by the rotating switch, which switches on and off the various parts of the system at the proper instant directly or by relays, and connects the coulometers to the output one by one in succession.

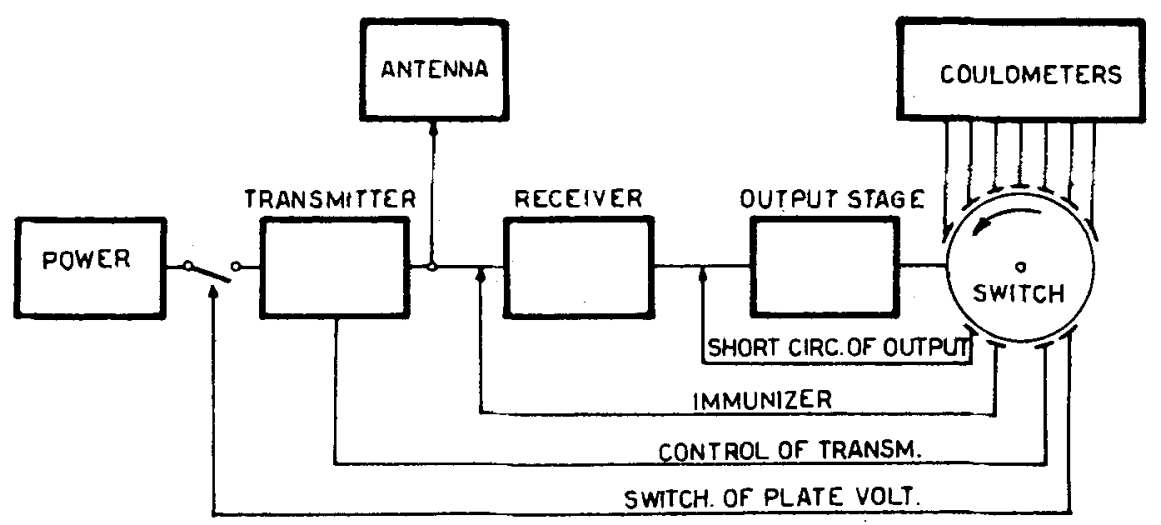

Fig. 2.

THE ANTENNA SYSTEM.

Concerning the success of the experiment, the energy concentration performed by the antenna system plays an important part, and it is most essential to increase this factor as far as possible. At 2,5 m's wavelength focusing can be achieved by applying a system of dipole-antennas.

For our experiment such a system was designed by $E$. Istvánffy. The wiring of the aerial system is shown on Fig. 3. The system consists of 36 dipole-antennas, which are fed in the manner that the single antennas shou'd oscillate in phase. Each one has potential-node at its middle point, which is in galvanic contact with the supporting metallic rod. The supporting rods stand out at right angles from the plan of a huge iron framework by a quarter-wave-length, the framework itself forming a reflecting surface making the radiation unidirectional. The surface area of the framework is $8 \times 6 \mathrm{~m}^{2}$.

The iron framework was manufactured in the workshop of the Tungsram Research Laboratory according to the design of E. Istvánffy; J. Patak was in charge of the work. The plan of the framework can be rotated around a horizontal and a vertical axis driven by gears, so that it can be turned and fixed to any direction of the sky. (Fig. 4.) The antenna system is shown in Fig. 5. The directional pattern is shown in polar coordi- 


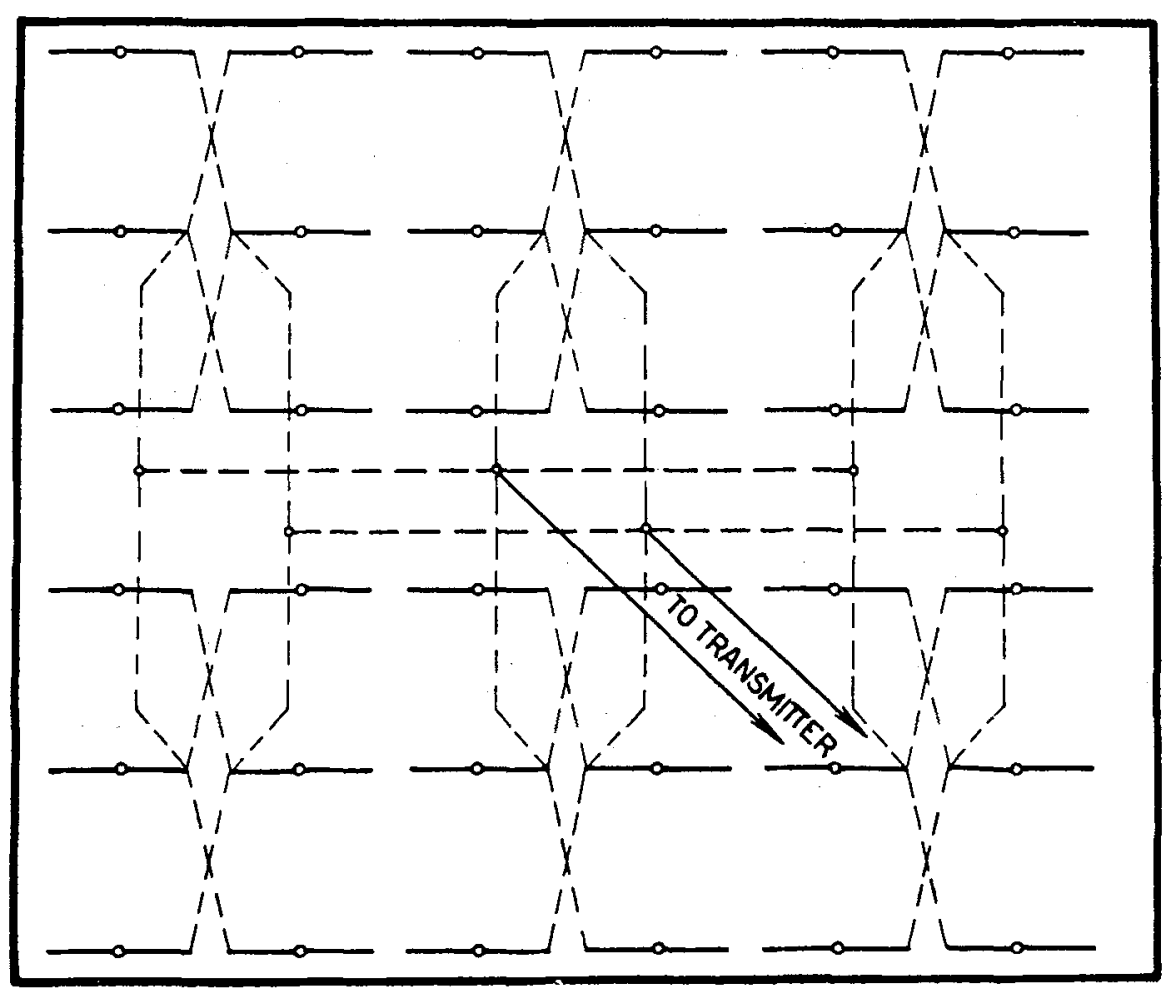

Fig. 3

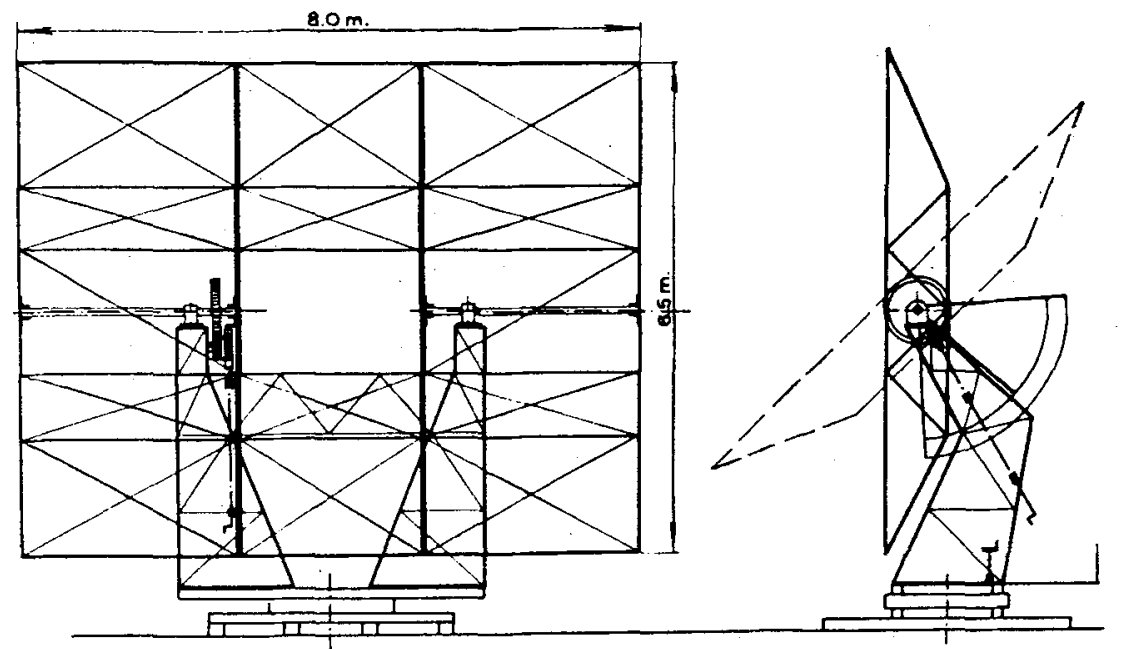

Fig. 4. 


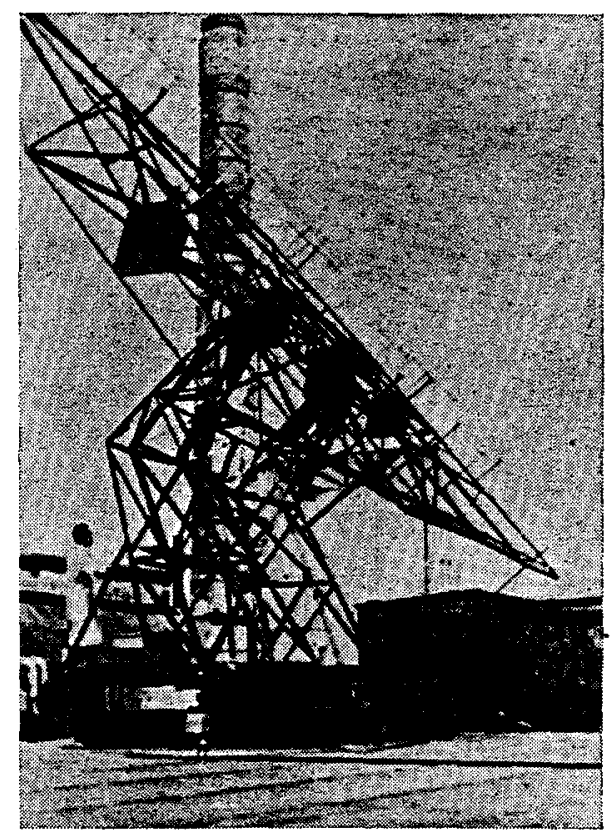

Fig. 5.
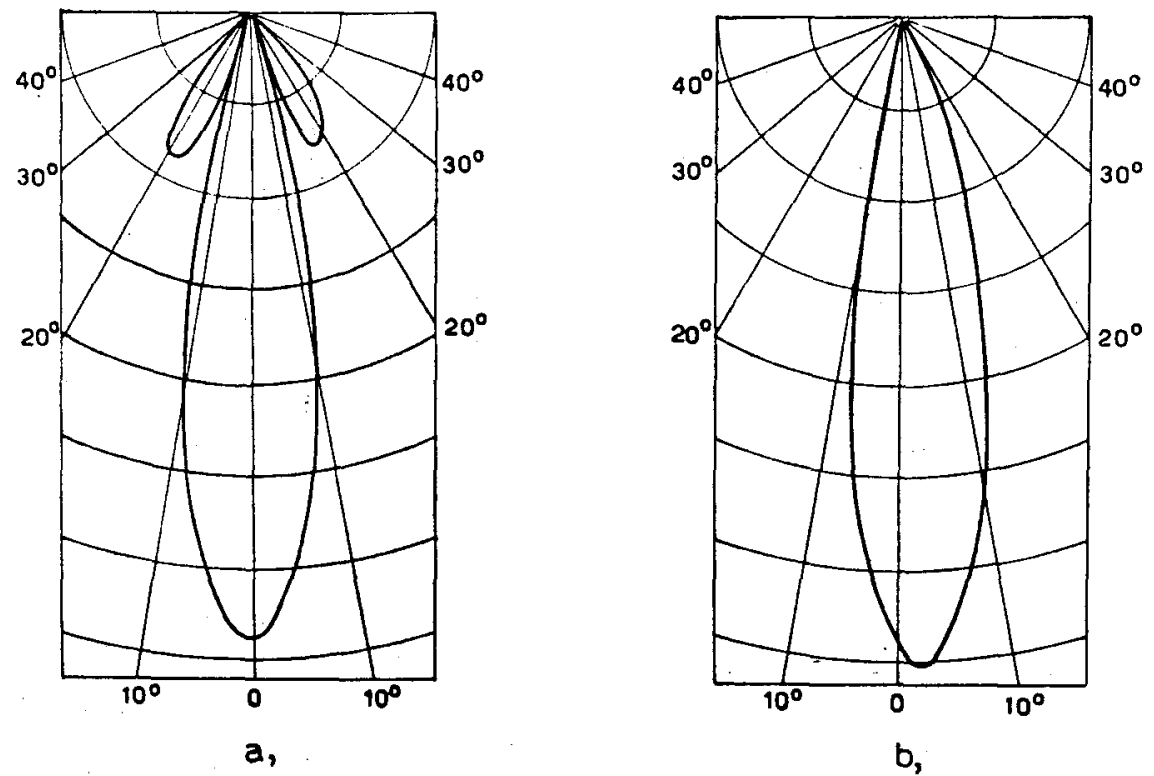

Fig. 6. 
nate-system, Fig. 6., $a$ being the horizontal, $b$ the vertical section. The factor of the increase of the field intensity on the electric axis - computed from the polar characteristics - is $\varrho \approx 9$.

\section{THE TRANSMITTER. (Fig. 7.)}

The transmitter originally manufactured by Standard was modified for operation with two OQQ 500/3000 Tungsram tubes, connected in push-pull. The plate resonant circuit is formed by a parallel Lechersystem, the leads having large cross section in order to reduce damping. Both grid and cathode circuits are tuned by Lecher-systems. The transmitter is controlled in the following way: the grid biased to $-2000 \mathrm{~V}$ is grounded by the rotating switch for $0,06 \mathrm{sec}$ in each 3 sec's interval,

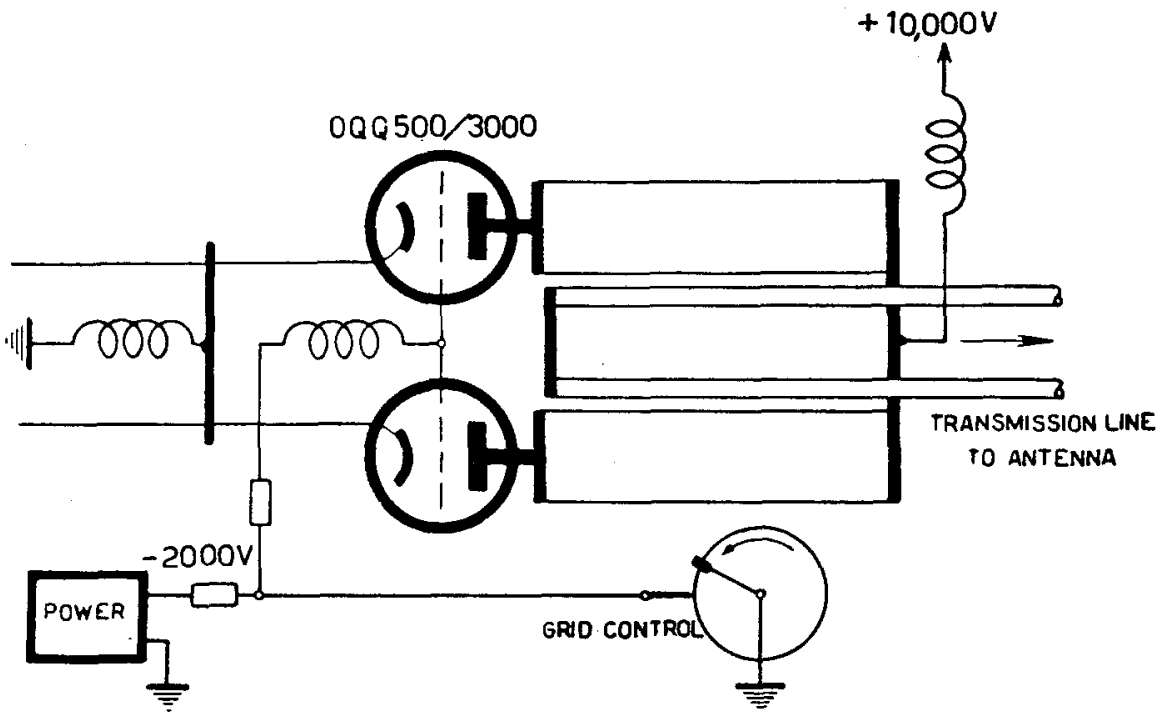

Fig. 7.

giving an impulse of $0,06 \mathrm{sec}$ 's duration. The plate voltage is $6000-10.000 \mathrm{~V}$, and as the tubes are not constructed for such a high voltage the rotating switch also provides for turning off the plate voltage during the long pauses between two successive impulses.

The average plate-current during the impulse is about $2 \mathrm{~A}, \mathrm{i}$. e. the input power is $\mathrm{I} 2 \mathrm{~kW}$ at a plate-voltage of $6000 \mathrm{~V}$. 
The efficiency of the tubes in the present arrangement is about $30-40 \% \mathrm{i}$. e. the $\mathrm{h}$. f. power is about $3-4 \mathrm{~kW}$. The decoupling of the oscillations takes place by a simple loop, formed by the short-circuited end of the transmission line.

\section{THE RECEIVER.}

A block diagram of the receiver closing with the demodulator is shown on Fig. 8. High frequency oscillations are amplified by the first stage (Tube EFF50), and then the first converter stage (Tube EFF50) transforms the frequency from $120 \mathrm{Mc}$. p. s. into $4,3 \mathrm{Mc}$. p.s. On the block diagram also a second converter stage is to be seen, producing the 2,7 Mc. p. s. intermediate-frequency, following the detector stage. The matching of the receiver to the transmission line is arrived at through small capaci-

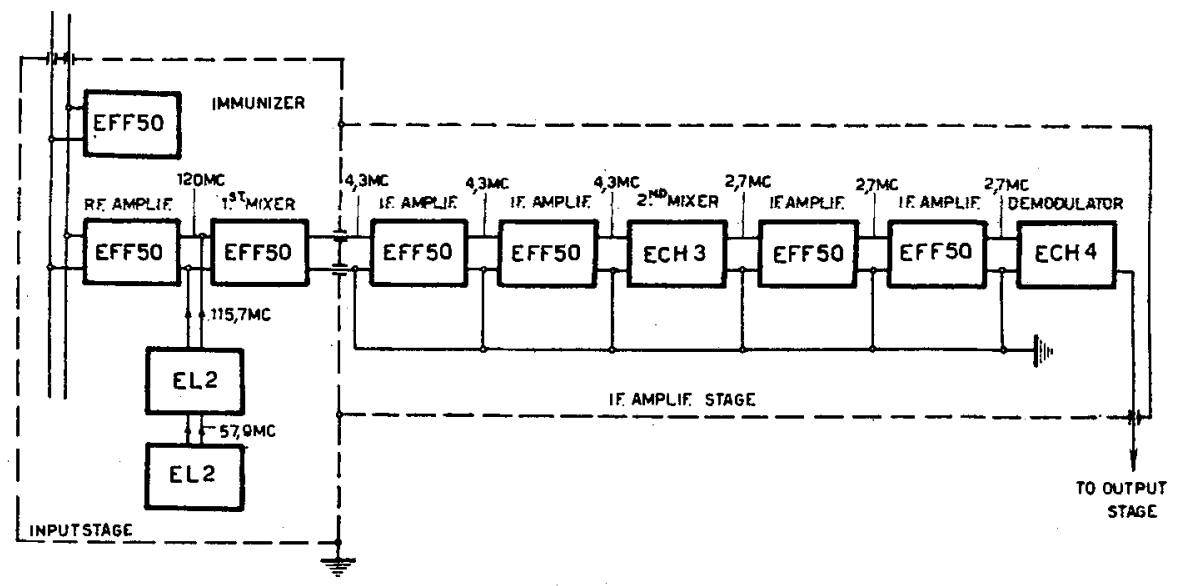

Fig. 8.

ties by a tuned Lecher-system, being an impedance-transformer. The amplification factor of the first amplifier is 3 , that of the frequency transformer stage 2. The band-width of the receiver is $200 \mathrm{kc}$. p. s.

During the transmission of the impulse, the receiver gets a voltagepulse of about rooo $\mathrm{V}$, which might damage the first stage. To avoid this, an immunizing device had to be applied. At the beginning an $E_{5 F}$ type tube connected as a diode was used for this purpose. As, however, this tube was also often damaged, a short-circuiting lever was introduced, operated shortly before the transmission of the signal by an electromagnet, controlled by the rotating switch. 
The band-width reducing output stage is shown schematically in Fig. 9. The first low frequency filter unit is placed in the receiver, the two low frequency amplifier stages are mounted in the next room in a shielded "cage" together with the coulometers and the rotating switch. Contact is maintined through a shielded cable. The amplification characteristic is shown in Fig. Io. Practically frequencies between 3-50 c. p. s. can pass the output stage which means a reduction of the noise level compared to that of the demodulator by a factor of about roo. Meanwhile the reflected

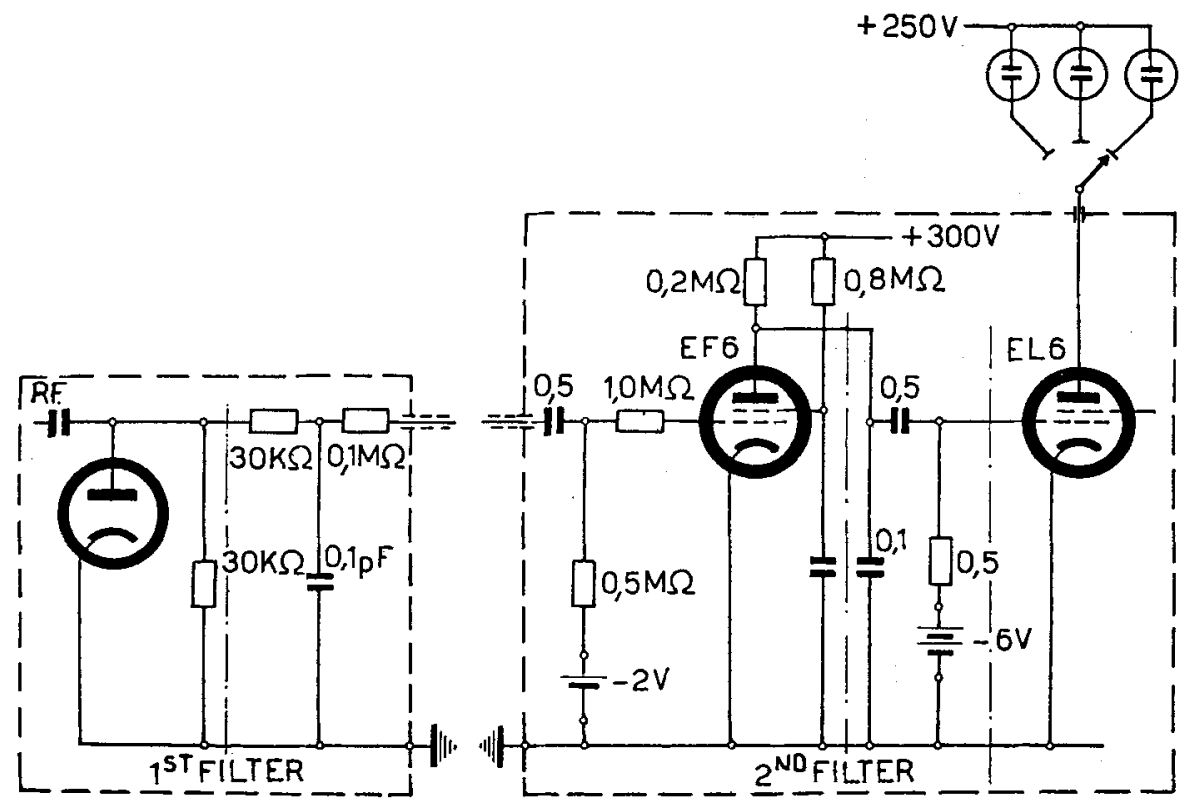

Fig. 9.

signal, having the shape of the transmitted signal, owing to its $0,06 \mathrm{sec}$ period, passes the output stage almost with its original amplitude, as its Fourier-spectrum falls practically within the frequency band of the output stage.

Immunizing of the output stage during the transmission period is also necessary, as in spite of immunizing the first stage of the sensitive receiver the output stage still receives such an impulse as would change the operational points of the tubes for a long time - owing to the large time constants of its circuits. For this reason the grid leak resistance of the tube EF 6 is short-circuited by a special magnetic relay for the period of the transmission, as shown in Fig. I2. 

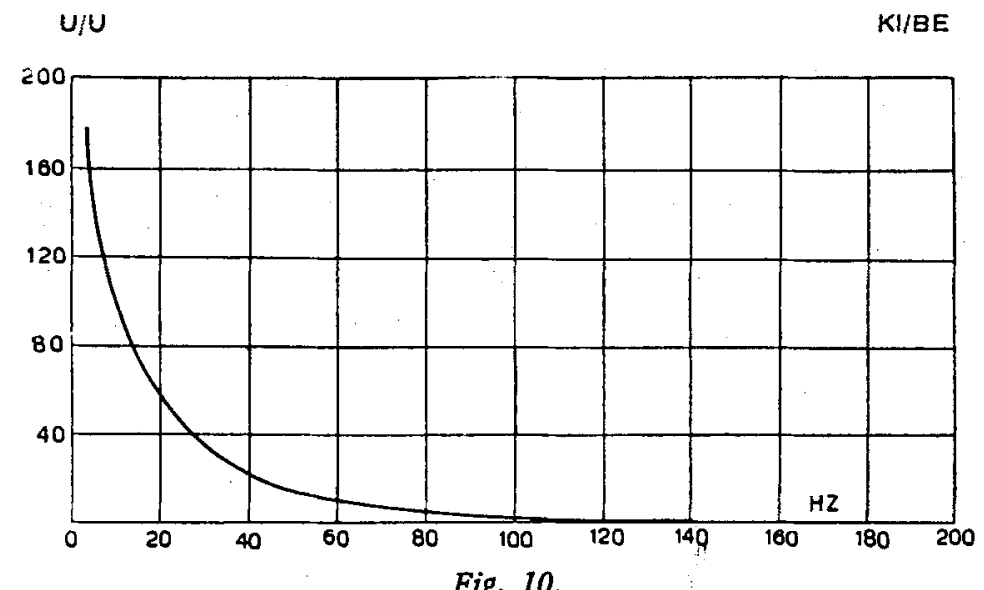

Fig. 10.

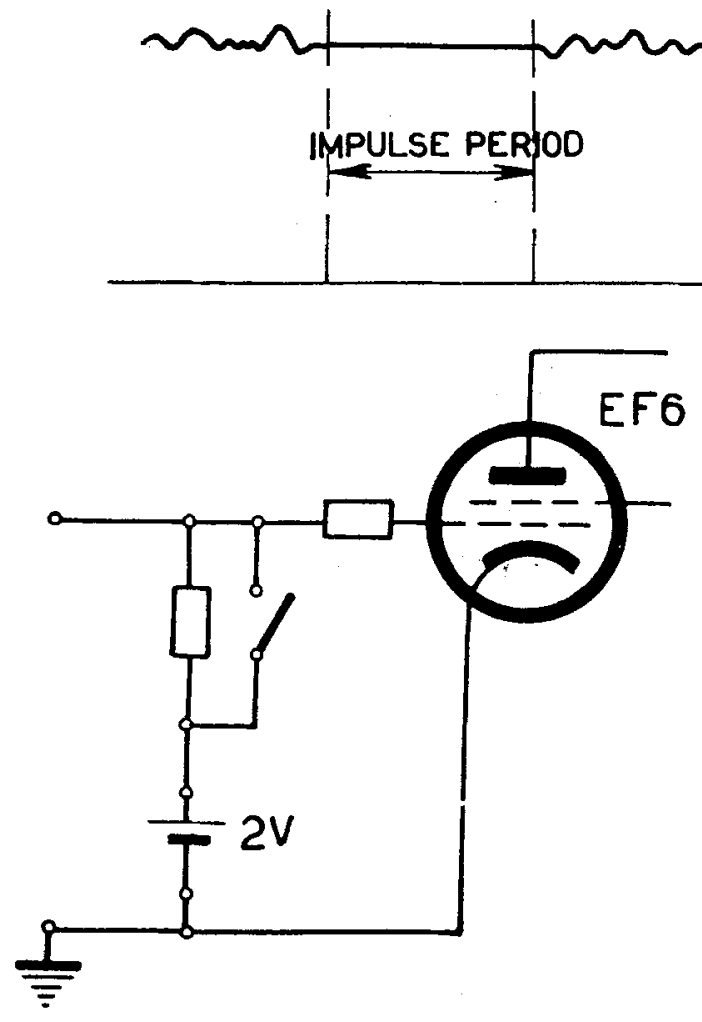

Fig. 11 . 


\section{THE ROTATING SWITCH.}

The schematic diagram of the rotating switch is shown in Fig. I2. The contacts on a fixed disc are touched by the contacts of the rotating arm in succession.

The rotating arm is driven by a synchronous motor by gears designed in such a manner that at the $50 \mathrm{c} . \mathrm{p}$. s. frequency of the mains, one revolution of the arm takes $3 \mathrm{sec}$. A few percent fluctuation of the alternating current's frequency often caused trouble as the reflected signal

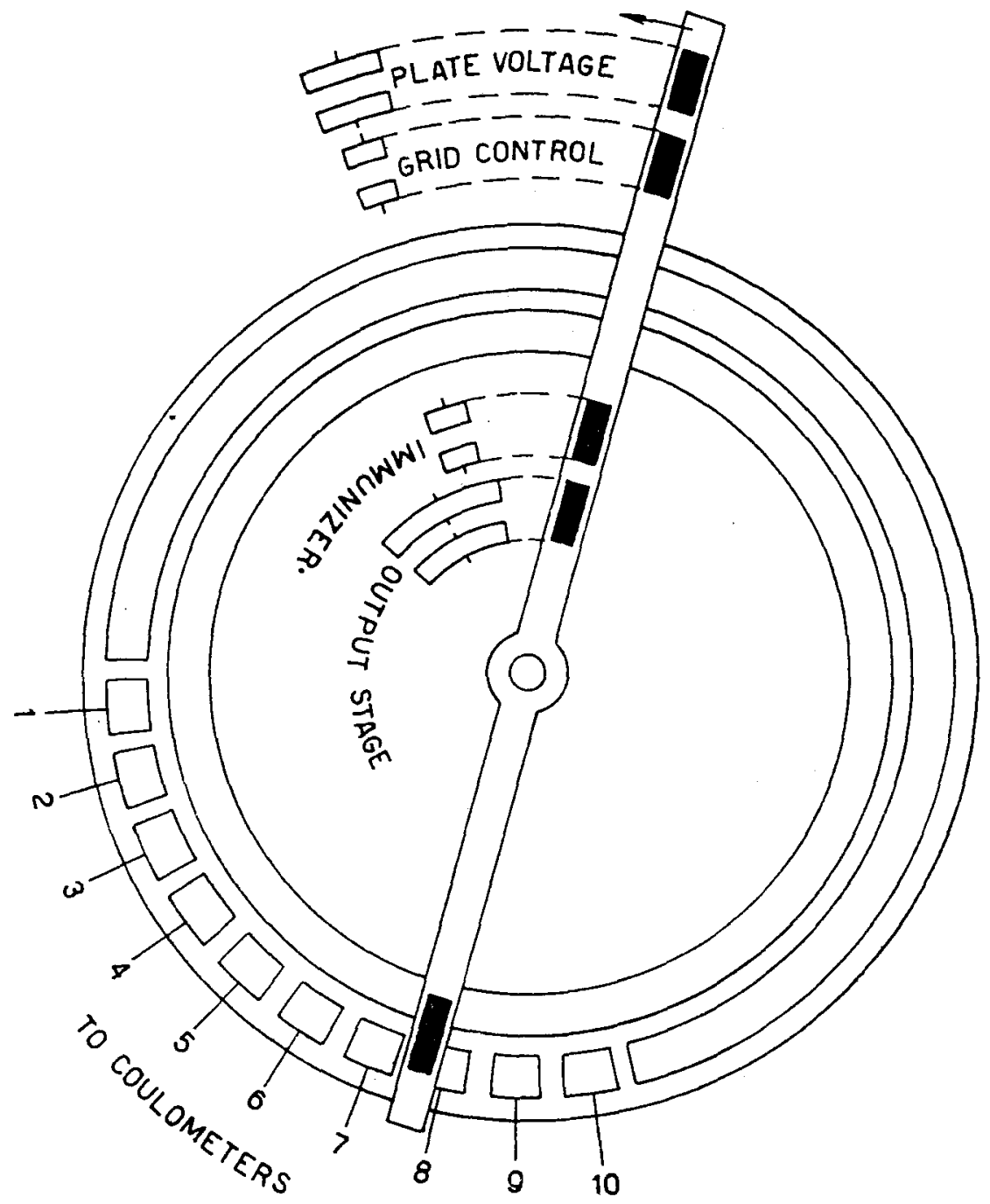

Fig. 12. 
can slip onto a neighbouring coulometer during an experiment. For this reason the frequency was controlled continuously.

The switch was designed by $A$. Budincsevics, and manufactured in the workshop of the Reseach Laboratory, J. Patak was in charge of this work.

On the arrival of the reflected signal the coulometers are switched on and however small sudden changes in voltage, they are enough to disturb the receiver. For this reason the rotating switch together with the output stage and the coulometers had to be put into a shielded cage and provided with a most careful filtering before the leads leaving the cage. These precautions were necessary, for these disturbances are not averaged out, being of periodical character.

\section{THE COULOMETERS}

are shown in Fig. 13. All coulometers communicate with the same reservoir on top, containing the common positive electrode. Hydrogen gas formed on the negative electrodes is gathered in the capillaries, and their quantity can be directly read. The coulometers were made by E. Várbiró, glass-blower of the Laboratory.

A KOH-solution of $30 \%$ concentration was found to be most favourable electrolyte, having such a kind of capillarity that very fine bubbles are formed in it, which rise in the I $\mathrm{mm}$ diameter capillary without interrupting the liquid column. Oxyhydrogen-coulometers, tested previously,

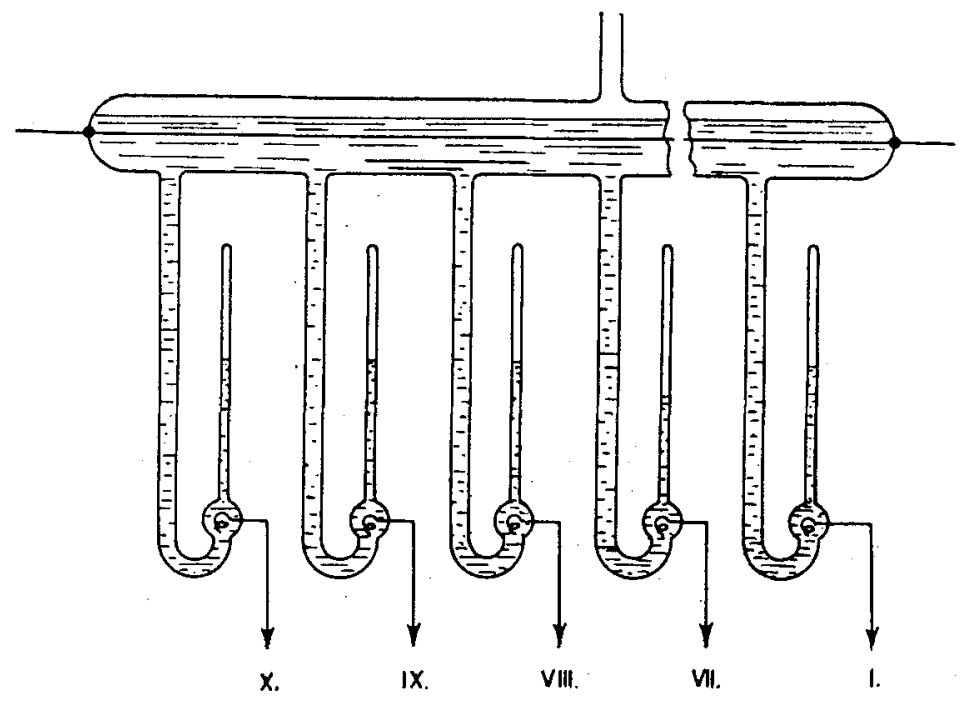

Fig. 13 
gave no reproducible results, owing to the presence of nascent oxygen and the oxydation of the electrodes. In the above case, when no oxygen is formed on the measuring electrodes, the measurements can be reproduced within I\% accuracy. In the experiments the cou ometers led a maximum current of $60 \mathrm{~mA}$ intensity, the velocity of the motion of the liquid's $\mathrm{m}$ ?niscus was $4-6 \mathrm{~mm} / \mathrm{mA} . / \mathrm{min}$. This value, however, can be varied practically as necessary, by reducing the gas pressure in the top reservoir, obtaining in this. way an apparatus, where one can choose the scale of measurements similarly to that of the MacLeod pressure gauge.

\section{THE EXPERIMENTS AND RESULTS,}

As soon as the equipment had been completed, in January 1946 , the experiments were begun.

During the working hours of the plant, electrical disturbances are too great, so that measurements and preparations (tuning, etc.) can be carried out only in late afternoon hours and at night. For this reason no measurements can be taken about new-moon, when the Moon is very near to the Sun.

Knowing the electrical axis of the antenna system, in fair weather it can be directed towards the Moon visually, and in cloudy weather, the right direction was found by the determination of the Moon's position on the sky based on astronomical data, received from the Konkoly Observatory. ${ }^{7}$ The calculations were made by $T$. Horváth and L. Takács.

The apparent displacement of the Moon is about $15^{0}$ per hour, so it can be followed easily during the experiment. When pointing at the Moon, a few degrees of deviation may be allowed, owing to the directional pattern of the antenna system.

Tuning the transmitter and the receiver is accomplished either by terrestrial reflections, or by a small auxiliary transmitter, by which tuning can be controlled.

The experiment consists of two kinds of measurements carried out alternately: one performed with the antenna system pointing towards the Moon, and the other with the antenna system turned away ("blind" measurement) both having a period of about $30 \mathrm{~min}$.

The measurements are preceded by the testing of the coulometers. Their resistances and the length of the contacts belonging to each one

7 We are indebted to Dr. L. Detre, Director of the Observatory, for giving us the necessary data. 
are a little different. That is why it is necessary to carry out measurements during which the receiver is out of operation, and only the uncontrolled current of the output stage flows through the coulometers. The next stage of testing is accomplished with the receiver in operation, and the transmitter out of operation. In this case only the noise of the receiver passes through the coulometers. The longer the measurements the better the agreement between the results of these tests, because the coulometer averages out the statistical fluctuations of the receiver. If the measurements take a few minutes, practically no difference is to be found between the results received by the two different methods, i. e. after a few minutes the statistical error is less than the error in reading the position of the meniscus in the capillary (parallax).

After these operations the Moon-experiment has to be continued until the signal on one distinguished coulometer exceeds the errors of the measurement. This measurement is followed by the "blind" experiment.

The measurements were carried out during several nights by $D r . K$. Simonyi, Dr. J. Pócza, Z. Bodó, T. Horváth, J. Csiki, and L. Takács, under the direction of Dr. G. Papp. Two observers were present at each measurement.

\begin{tabular}{|c|c|c|c|c|c|c|c|c|c|c|c|}
\hline & I & II & III & IV & $\mathrm{v}$ & VI & VII & VIII & IX & $\mathrm{x}$ & Note \\
\hline Moon & - & - & - & - & - & 110 & 100 & 100 & 92 & 96 & \multirow{3}{*}{$\begin{array}{l}\text { Experiment on } \\
6.2 .46 \text {. }\end{array}$} \\
\hline Blind & - & - & - & - & 一 & 110 & 96 & 101 & 93 & 97 & \\
\hline Difference & - & 一 & 一 & 一 & - & 0 & +4 & -1 & -1 & -1 & \\
\hline Moon & 96.5 & 92.5 & 90.9 & 103.5 & 97.5 & 103.3 & 111.2 & 98.8 & 100.2 & 106.4 & \multirow{3}{*}{$\begin{array}{l}\text { Experiment on } \\
\text { 8. 5. }+6 .\end{array}$} \\
\hline Blind & 97,1 & 93.3 & 91.1 & 103.2 & 97.0 & 98.9 & 110.8 & 100.7 & 101.7 & 105.6 & \\
\hline Difference & -0.6 & -0.7 & -0.2 & +0.2 & +0.5 & +4.4 & +0.4 & -1.9 & -1.5 & +0.8 & \\
\hline Artif. refl. & 72.1 & 79.8 & $8 \div .2$ & 93.0 & 90.0 & 93.8 & 100.0 & 162.8 & 128.8 & 97.6 & Signal/noise $\sim 1$ \\
\hline Blind & 95.1. & 92.5 & 88.1 & 102.9 & 100.3 & 105.4 & 112.3 & 112.3 & 88.1 & 109.0 & \\
\hline Difference & -13 & -12.7 & -5.9 & -9.9 & -10.3 & -11.8 & -12.3 & +50.5 & +40.7 & -11.4 & \\
\hline Artif. refl. & $9 \mathrm{I.3}$ & 88.9 & 89.2 & 97.7 & 98.3 & 107.5 & 105.6 & 119.7 & 98.6 & .102 .8 & Signal/noise $\sim \frac{1}{5}$ \\
\hline Blind & 9.0 & 91.6 & 90.1 & 99.3 & 98.7 & 108.8 & 108.6 & 111.5 & 95.0 & 104,8 & \\
\hline Difference & -2.7 & -2.7 & -0.9 & -1.6 & -0.4 & -1.3 & -3.0 & +8.2 & +3.6 & -2.0 & \\
\hline
\end{tabular}

Table 1 .

The result of a few series of measurements are shown in Table I. It can be seen that in case of the measurement carried out on the 6th February, I946, the readings on Coulometer VII. were $4 \%$ higher in Moon-experiment, than in blind experiment, although the error is under $\mathrm{r} \%$. According to the measurement carried out on the 8th May, 1946 Coulometer VI, gave a reading $4, \mathrm{r} \%$ higher in the Moon-experiment, than in blind experiment, etc. 
When evaluating the results, it must be also considered that the coulometers measure the unmodulated component of the plate-current of the last tube too. The easiest way to get information about the amplitude of the received signal is to compare it with the noise of the receiver, which can be expressed in microvolts.

To measure the signal/noise ratio Dr. G. Papp developed the method of "artificial reflection"; its principle can be seen on Fig. I4. Taking one of the coulometers (No. VIII. in Fig. I4.), at the instant

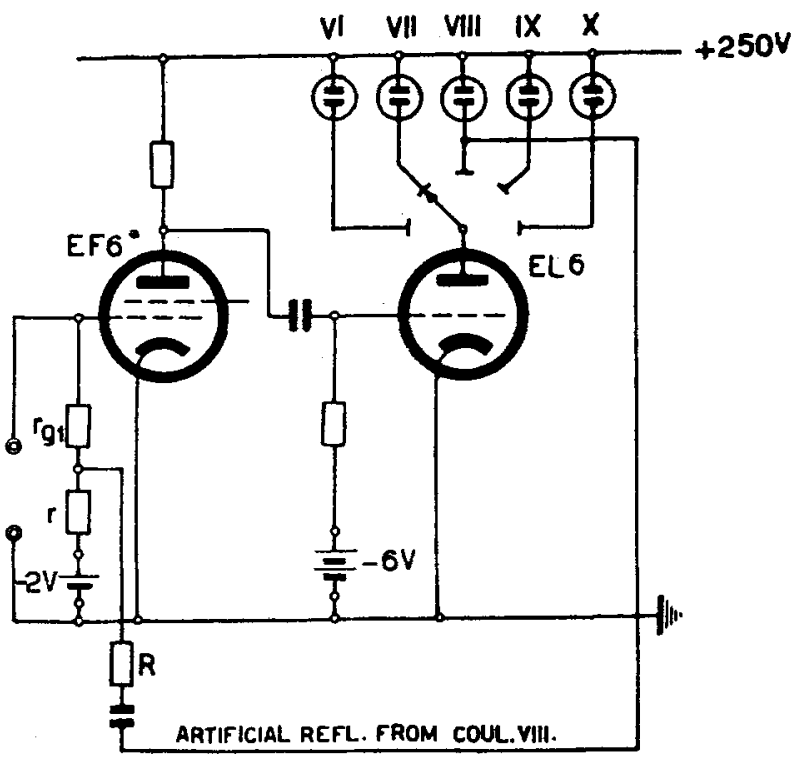

Fig. 14 .

when it is switched on, the voltage of its cathode is reduced by about $20 \mathrm{~V}$. A definite fraction of this voltage is divided on a potentiometer consisting of the resistances $r$ and $R$ and fed back to the grid of the tube EF 6 , by which it is amplified and so increases the current through Coulometer VIII. The conditions are the same as if Coulometer VIII. had received a reflection from the Moon. By varying the resistances $r$ and $R$ a known and the required signal/noise ratio can be produced, where "signal" now means the amplitude of the "artificial reflection".

Such measurements have shown that a $4 \%$ effect on a coulometer is equivalent with a signal/noise $=\mathrm{r} / \mathrm{ro}, \mathrm{i}$. $\mathrm{e}$, the amplitude of the reflected signal from the Moon in the experiments above described is $I /$ ro of the noise of the receiver, computed for the output, as theoretically expected. 
(See p. 5.) The fact that this minute signal can be observed or even estimated with the aid of our equipment, is exclusively due to the method of coulometers.

We can compare our results with those of the American investigators De Witt and G. Valley as communicated in "Electronics". ${ }^{8}$ These authors, using a receiver of $0,0 \mathrm{I} \mu \mathrm{V}$ sensitivity and having a band width of $50 \mathrm{c} . \mathrm{p} . \mathrm{s}$., achieve a signal/noise ratio of about 3 .

The power of their transmitter is about the same as that of the ours. The factor $\nu$ characterising the sensitivity of their receiver seems to be more favourable than that we could realise, and we estimated it to be $40 \%$ smaller. Their antenna system consists of 64 dipole-antennas, which means about $70 \%$ gain compared to our case. Considering all these circumstances, our theoretical computation gives a signal/noise $\approx 9$ for their experiments, which fully agrees with their results.

The most important difference between the two equipments is, that the reduction of the band width is accomplished in their apparatus before the demodulator stage, the theoretical advantage of which was described on p. 5. The American radar practice, having developed to a very high standard, could solve this difficult problem, the expense of which we could not afford.

Neither the.American nor our results are accurate enough for more than the purpose of estimating the order of magnitude of the numerical value of factors mentioned in the introduction, such as reflection coefficient of the Moon's surface, absorption and dispersion in the ionosphere. It is, however, indisputable that the order of magnitude is given by the experiments as expected.

It is significant that electromagnetic waves of $2,5 \mathrm{~m}$ wavelength can pass through the ionosphere. This fact may receive practical importance in interplanetary navigation and in spreading reflected microwaves over the whole surface of the Earth.

The success of our Moon-experiment adds the most direct evidence to that which confirms the validity of the Copernican view. There had been a number of observed phenomena from which it followed that the Moon is a material body situated at a well-defined distance from the Earth. This statement is demonstrated ad oculos by the Moon-experiment: we can "touch" the Moon by a pencil of electromagnetic waves whenever we wish. The transit time of the waves and the material properties of the Moon as shown by the reflection agree with the expectations.

8 Electronics, April, I946., p. 92. 
The fact that American investigators carried out successful Moonexperiments without the method of cumulation, does not diminish the value of using coulometers. In the future the coulometers (or other method of cumulation) can be used for more exact measurements without increasing the transmitted energy substantially, or the measurements can be extended to other celestial objects too. In this field the best results would be yielded by a combination of highly developed American technique with our method of cumulation. 